\title{
Real-World Evidence for Control of Chronic Migraine Patients Receiving CGRP Monoclonal Antibody Therapy Added to OnabotulinumtoxinA: A Retrospective Chart Review
}

\author{
Andrew M. Blumenfeld - Benjamin M. Frishberg • Jack D. Schim • \\ Ashley Iannone - Gary Schneider • Larisa Yedigarova • \\ Aubrey Manack Adams
}

Received: December 18, 2020 / Accepted: April 1, 2021 / Published online: April 21, 2021

(C) The Author(s) 2021, corrected publication 2021

\section{ABSTRACT}

Introduction: Combination use of onabotulinumtoxinA and calcitonin gene-related peptide (CGRP) monoclonal antibodies (mAbs) has the potential to be more effective than either therapy alone for migraine prevention.

Methods: This retrospective, longitudinal chart review included adults with chronic migraine treated at one clinical site with $\geq 2$ consecutive cycles of onabotulinumtoxinA and $\geq 1$ month of subsequent combination treatment with CGRP mAbs. Charts at time of $\mathrm{mAb}$ prescription (baseline) and up to four visits $\sim 3,6,9$, and 12 months post-baseline were reviewed for safety, tolerability, and outcome measures (monthly headache days [MHDs],

Ashley Iannone, Gary Schneider, and Larisa Yedigarova: Former employees.

Supplementary Information The online version contains supplementary material available at https:// doi.org/10.1007/s40122-021-00264-x.

A. M. Blumenfeld $(\bowtie) \cdot$ B. M. Frishberg · J. D. Schim Headache Center of Southern California, The

Neurology Center, 6010 Hidden Valley Road, Suite 200, Carlsbad, CA 92011, USA

e-mail: blumenfeld@neurocenter.com

A. Iannone - G. Schneider

ICON Plc, Boston, MA, USA

L. Yedigarova $\cdot$ A. Manack Adams

Allergan, an AbbVie Company, Irvine, CA, USA headache intensity, and migraine-related disability [MIDAS]).

Results: Of 300 charts reviewed, 257 patients met eligibility criteria (mean age: 50 years; $82 \%$ women). Average headache frequency was 21.5 MHDs before initiation of onabotulinumtoxinA and 12.1 MHDs before adding CGRP $\mathrm{mAb}$ therapy. Prescribed mAbs were erenumab $(78 \%)$, fremanezumab $(6 \%)$, and galcanezumab (16\%). Over the entire study, patients discontinued CGRP mAb more frequently than onabotulinumtoxinA (23 vs. 3\%). Adverse events occurred in $28 \%$ of patients, most commonly constipation (9\%). Compared with onabotulinumtoxinA alone (baseline), MHDs decreased significantly at all visits (mean decrease: $3.5-4.0$ MHDs over $\sim 6-12$ months of combination treatment); $45.1 \%$ of patients had clinically meaningful improvement in migraine-related disability ( $\geq 5$-point reduction in MIDAS score) after $\sim 6$ months.

Conclusions: In this real-world study, combination treatment with onabotulinumtoxinA and CGRP mAbs was well tolerated, with no new safety signals identified, and was associated with additional clinically meaningful benefits. More real-world and controlled trials should be considered to further assess safety and potential benefits of combination treatment.

Keywords: CGRP receptor; Chronic daily headache; Chronic headache; Combination 
therapy; Migraine headache; Preventive treatment; Type A botulinum toxins

\section{Key Summary Points}

\section{Why carry out this study?}

A multimodal treatment approach that layers treatments targeting different physiological pathways involved in migraine may improve outcomes in patients with chronic migraine.

OnabotulinumtoxinA and calcitonin gene-related peptide monoclonal antibodies (CGRP mAbs) act through different physiological mechanisms and are both effective and well tolerated when administered individually for the preventive treatment of migraine.

We collected real-world data from chronic migraine patients who received CGRP $\mathrm{mAb}$ treatment added to onabotulinumtoxinA treatment to investigate the safety, tolerability, and potential benefits of this combination treatment.

\section{What was learned from the study?}

Adding a CGRP mAb to onabotulinumtoxinA was safe and well tolerated, with no new safety signals identified.

Combination treatment significantly decreased monthly headache frequency and migraine-related disability compared with onabotulinumtoxinA alone.

\section{DIGITAL FEATURES}

This article is published with digital features, including a summary slide and video abstract, to facilitate understanding of the article. To view digital features for this article go to https:// doi.org/10.6084/m9.figshare.14322125.

\section{INTRODUCTION}

Individuals with chronic migraine $(\mathrm{CM} ; \geq 15$ monthly headache days [MHDs]) [1] experience greater severity of migraine-associated disability [2] and greater prevalence of comorbid conditions (e.g., other chronic pain disorders, anxiety, depression) than those with less frequent migraine attacks $(<15$ MHDs) [3, 4]. CM can greatly impair daily activities and quality of life, leading to substantial burden to the family and society $[5,6]$. Given the high burden of CM, a multimodal management approach layering treatments that target different pathways involved in migraine pathophysiology may improve outcomes.

OnabotulinumtoxinA has been approved for CM prevention since 2010 and is well tolerated, with proven efficacy across multiple clinical and real-world studies [7-11]. For CM, onabotulinumtoxinA is injected into specific head and neck muscles containing sensory neuron endings with cell bodies located in trigeminal and cervical ganglia [12]. By inhibiting activation of these sensory inputs, onabotulinumtoxinA reduces the magnitude of pain signaling in the brain and prevents activation and sensitization of central neurons thought to be involved in migraine chronification. A key mechanism of action of onabotulinumtoxinA is the attenuation of the release of neuropeptides and neurotransmitters into the synapse, and the insertion of receptors and ion channels into the nerve terminal membrane, which modulates the activation of receptors implicated in migraine pathophysiology, including glutamate, substance $P$, and calcitonin gene-related peptide (CGRP) [12]. Attenuation of CGRP release from peripheral nerve terminals of meningeal and trigeminal nociceptors plays a key role in the effect of onabotulinumtoxinA on migraine [12-15].

Direct inhibition of CGRP pathways has emerged as a targeted approach for migraine prevention [16]. In 2018, three subcutaneously injected monoclonal antibodies (mAbs) directed against CGRP or its receptor (i.e., erenumab, fremanezumab, and galcanezumab) were approved by the US Food and Drug 
Administration (FDA) for migraine prevention [17-19]. Erenumab targets the CGRP receptor, whereas galcanezumab and fremanezumab prevent CGRP ligand binding to the CGRP receptor.

Combination treatment with mechanistically distinct preventive therapies could have additive or synergistic effects in migraine prevention [20], as supported by preclinical studies demonstrating that onabotulinumtoxinA and CGRP mAbs prevent activation of different types of pain fibers. CGRP mAbs mainly prevent activation of thinly myelinated A $\delta$-fibers [21], and onabotulinumtoxinA prevents activation of myelinated C-fibers [22]. There have been several clinical reports of adding CGRP mAb therapy to onabotulinumtoxinA treatment in realworld settings [23-30], many of which have evidence of clinical benefits of combination treatment [23-25, 28, 30].

The treatment of migraine in patients with $\mathrm{CM}$ who experienced previous prophylactic therapy failure is a challenge. While patients with CM often benefit from onabotulinumtoxinA treatment, they may continue to experience migraine attacks at a frequency that meets criteria for receiving additional preventive treatments. Based on its distinct mechanism of action, adding a CGRP $\mathrm{mAb}$ to ongoing treatment with onabotulinumtoxinA may result in additional improvement. According to a position statement from the American Headache Society (AHS) in 2019, CGRP mAb therapy may be added to one or more established treatments based on clinical judgment [31]. As long as the risk of drug-antibody interactions is considered minimal or nonexistent, AHS supports adding a $\mathrm{mAb}$ to the existing regimen without making other changes until the effectiveness of the antibody therapy is determined [31]. Regarding the preventive treatment paradigm, CGRP mAb therapy may be appropriate for and initiated in adults with migraine who have had inadequate tolerability or response to a 6-week trial of at least two other preventives with established efficacy and 4-7 monthly headache days (MHDs) characterized as at least moderate migraine-related disability, 8-14 MHDs with any level of disability, and 15 or more MHDs (CM) and an inability to tolerate or inadequate response to a minimum of two quarterly injections (6 months) of onabotulinumtoxinA. These recommendations are consistent with how experts treat their patients in clinical practice.

To date, no randomized controlled trials have evaluated the safety and efficacy of combination treatment with onabotulinumtoxinA and CGRP $\mathrm{mAb}$ for migraine prevention. This study collected real-world data from the medical records of patients treated at a single clinical site to gain improved understanding of the safety, tolerability, and potential benefits of adding CGRP $\mathrm{mAb}$ to onabotulinumtoxinA treatment. Potential benefits of combination treatment were based on effectiveness assessments that are in the patient charts and are widely used and generally recognized as being reliable, accurate, and relevant to migraine.

\section{METHODS}

\section{Study Design}

This retrospective, longitudinal chart review included adults (aged $\geq 18$ years) with $\mathrm{CM}$ treated at The Neurology Center of Southern California (San Diego County, CA) between October 1, 2018, and November 1, 2019. The center is a private-practice headache center that primarily focuses on patients with $\mathrm{CM}$. The practice uses an electronic medical records system that allowed for the capture of data across the whole clinic. Site staff prepared de-identified extracts of charts from patients with a physician's diagnosis of CM. The index date (alternatively referred to as index visit) was defined as the initiation of combination onabotulinumtoxinA and CGRP mAb therapy. Included patients were required to have at least two consecutive onabotulinumtoxinA treatment cycles without concomitant CGRP mAb therapy during the 8-month qualification period prior to the index date, and $\geq 1$ month of subsequent combination treatment with onabotulinumtoxinA and CGRP mAb (Fig. 1). Baseline measure assessments included the index visit and reflected assessments of migraine characteristics over approximately 


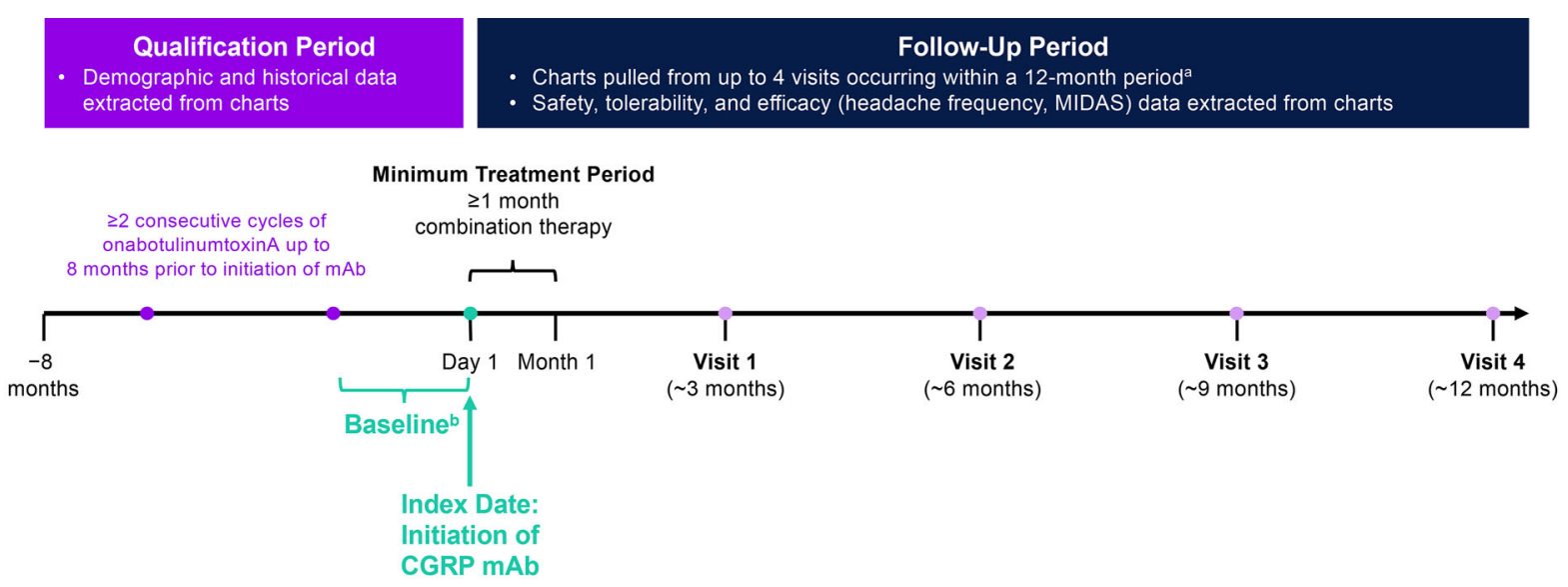

- OnabotulinumtoxinA treatment

- CGRP mAb treatment ${ }^{\circ}$

- Possible onabotulinumtoxinA treatment (per label) ${ }^{\mathrm{d}}$

Fig. 1 Study design. ${ }^{a}$ Not all patients had four visits or 12 months of data. ${ }^{b}$ Baseline assessments for outcome measures (e.g., headache day frequency, headache intensity, disability) were collected from the visit at which the CGRP $m A b$ was prescribed and reflect patient assessments during approximately 1-3 months prior to initiation of the CGRP mAb. ${ }^{~}{ }^{C G R P} \mathrm{mAbs}$ were self-administered by

1-3 months prior to the CGRP mAb prescription. To evaluate the incremental benefits of combination therapy, we collected, as available, the dates of migraine diagnosis, initiation of onabotulinumtoxinA, and headache frequency prior to onabotulinumtoxinA treatment prior to the 8-month qualification period.

No a priori power or sample size estimates were performed; this study used a convenience sample of approximately 300 patients based on available charts and adequate sample size to characterize the safety profile.

\section{Compliance with Ethics Guidelines}

The study was conducted in accordance with International Council for Harmonisation guidelines and local legal requirements, and complied with the ethical principles of the World Medical Assembly. The New England Independent Review Board approved the study protocol and case report form (CRF) before study initiation and determined that the study subcutaneous injection. Per label, erenumab and galcanezumab are administered once monthly, and fremanezumab is administered once every 3 months. ${ }^{\mathrm{d}}$ OnabotulinumtoxinA treatment is not always administered per label. CGRP calcitonin gene-related peptide, $m A b$ monoclonal antibody, MIDAS Migraine Disability Assessment

had minimal risk and met requirements for a consent waiver.

\section{Data Collection}

Data from de-identified charts were entered into an electronic CRF. Baseline demographic and clinical characteristics were recorded, as were index treatments received (i.e., type of CGRP $\mathrm{mAb}$ and dose), changes in treatment during follow-up (e.g., dose and/or brand), safety data, headache day frequency (per-patient self-report), headache intensity, migraine-related disability, headache impact, and depression (assessed using the Patient Health Questionnaire [PHQ-9]; moderate to severe depression was defined as PHQ-9 score $>9$ [32]) assessments from up to four follow-up visits. Visits generally coincided with clinic visits for onabotulinumtoxinA administration, which typically occur at 12- to 15 -week intervals. Visits $1,2,3$, and 4 occurred at approximately 3, 6, 9, and 12 months, respectively, after the initiation of combination treatment. 


\section{Safety and Tolerability}

Adverse events (AEs), discontinuations, and reasons for discontinuation were recorded for each visit. The CRF permitted input of up to five AEs/patient at each visit. This did not limit AE reporting, as no patients had more than five AEs at any visit.

\section{Outcome Assessments}

Due to variation in how headache frequency was recorded in patient charts and to reduce the risk of error during chart review, the CRF was constructed so that either a 30- or 90-day denominator could be used to report headache frequency. Ultimately, all headache day data collected using the 90-day denominator were converted to a 30-day (monthly) basis.

Headache intensity was captured on a scale of $0-10$, with scores of $0,1-4,5-7$, and $8-10$ indicating absent, mild, moderate, and severe headache intensity, respectively.

Migraine-related disability was captured on the Migraine Disability Assessment (MIDAS) questionnaire, a seven-item measure of headache-related disability in the previous 3 months [33]. MIDAS has five scored items assessing the total number of days migraine prevented or limited activities in the past 3 months, with higher total scores indicating greater disability. A five-point MIDAS score change is considered a clinically meaningful overall reduction [34].

Because of the exploratory nature of this retrospective study, it was unknown whether all measures could be collected in full. Therefore, the final analyses were based on measures available for the majority of patients. The sixitem Headache Impact Test was present in only 43.7 to $50.9 \%$ of patient charts across visits 1 to 4 , and the PHQ-9 was reported in only 23.4 to $34.6 \%$ of charts across post-baseline visits. Therefore, post-baseline data for these assessments have not been included in this report.

\section{Statistical Analysis}

Data were summarized using descriptive statistics. Mean changes from baseline in headache frequency, headache intensity, and MIDAS were calculated for each follow-up visit. In patients with available baseline and follow-up data, mean changes from baseline with corresponding 95\% confidence intervals (CIs) were calculated. The proportions of patients with any reduction in MHDs, $\geq 50 \%$ reduction in MHDs, $\geq 5$-point reduction in MIDAS score, and $\geq 30 \%$ reduction in MIDAS score compared with baseline, and corresponding 95\% CIs, were calculated. No adjustments to alpha were made to control for multiple comparisons. All CIs are therefore nominal.

This chart abstraction study was conducted using existing data collected under routine clinical practices for the purpose of patient care, not research. Missing values may have been the result of several circumstances (e.g., information was not applicable for the patient, or not available in the chart or in the chart extract prepared by the site). Not all patients contributed data to all four post-baseline visits. This may have been due to loss to follow-up, but could have also been due to the patient not yet completing a full year of combination therapy at the time of database closure, since patients were not required to have a full year of data to be included. Missing data values, regardless of reason, were not imputed given the exploratory nature of the study and real-world design.

\section{Study Cohorts}

The primary analysis cohort included all patients meeting study inclusion criteria. To best understand the applicability of findings, a sensitivity analysis was conducted in a cohort aligned to the AHS position statement's recommendation for when to initiate preventive treatment with a CGRP mAb [31] and included only patients with at least four MHDs at baseline and at least moderate headache-related disability (MIDAS score $>11$ or HIT-6 score $>50$; Fig. 2). The AHS position statement also requires an inability to tolerate (due to AEs) or inadequate response to a 6-week trial of at least two oral preventives with established efficacy. Failure of prior preventives was not verified in available chart data, although it is likely that 


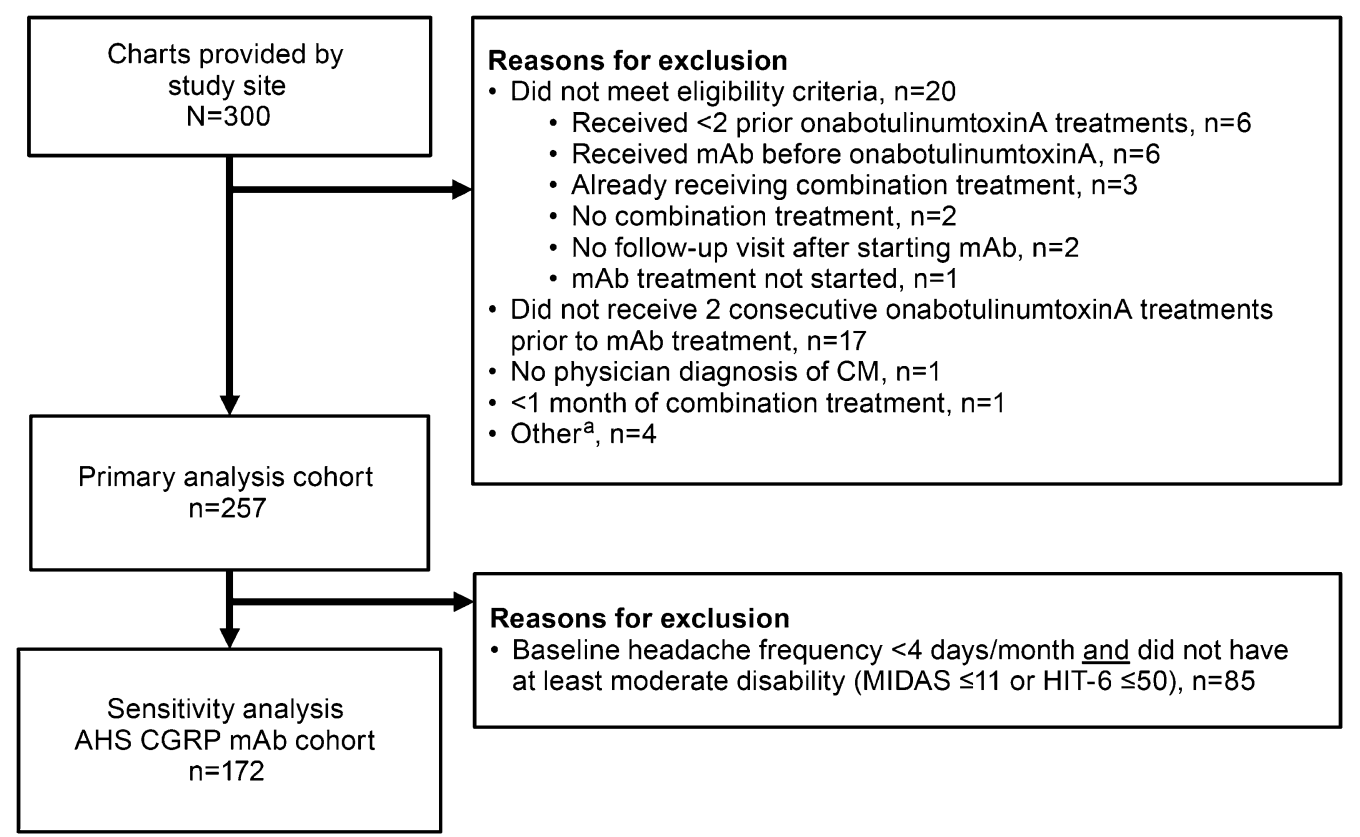

Fig. 2 Analysis cohorts for primary analysis and sensitivity analysis (AHS CGRP mAb cohort [31]). ${ }^{a}$ Other reasons for exclusion included negative time from migraine diagnosis to index date; negative time from first onabotulinumtoxin A injection to index date; headache frequency of 45/30 days recorded at index; and data

these patients did have such a history, as it would have likely been a payer requirement for onabotulinumtoxinA initiation.

\section{RESULTS}

Of 300 patient charts extracted, 257 patients met all inclusion criteria and comprised the primary analysis cohort (Fig. 2). Demographic and clinical characteristics are summarized in Table 1 . In the primary analysis cohort, mean age was 50 years (range, 21-94) and $82.1 \%$ were female. Concomitant use of other medications was recorded in $92.2 \%$ of patients at baseline, most commonly sumatriptan (20.7\%) and topiramate $(6.8 \%)$. Mean time since migraine diagnosis was 5.0 years and mean time since initiation of onabotulinumtoxinA was 3.8 years. Headache frequency was the only outcome measure that was consistently available in patients' charts for the time period prior to onabotulinumtoxinA initiation. The average available for only one visit (visit 4). AHS American Headache Society, CGRP calcitonin gene-related peptide, $C M$ chronic migraine, HIT-6 6-item Headache Impact Test, $m A b$ monoclonal antibody, MIDAS Migraine Disability Assessment

headache frequency was 21.5 MHDs before initiation of onabotulinumtoxinA and 12.1 MHDs before starting CGRP $\mathrm{mAb}$ as add-on therapy (Table 2). Mean change in headache frequency from pre-onabotulinumtoxinA to baseline (pre$\mathrm{mAb})$ was - 9.3 MHDs (95\% CI: - 10.7, - 7.9; $n=229)$. At baseline, $69.6 \% \quad(179 / 257)$ of patients had moderate to very severe disability, $112 / 138$ (81.2\%) had substantial to severe impact, and 14/69 (20.3\%) had moderate to severe depression (PHQ-9 score $>9$ ). Baseline clinical characteristics in the AHS [31] CGRP $\mathrm{mAb}$ cohort (sensitivity analysis) were similar to those in the primary analysis cohort (Tables 1 and 2).

\section{Combination Treatment Characteristics}

In the primary analysis cohort, the most commonly prescribed CGRP mAb was erenumab $(77.8 \%)$, with $52.9 \%$ of all patients receiving erenumab $70 \mathrm{mg}$ (Table 1). Mean interval 
Table 1 Baseline patient characteristics

\begin{tabular}{|c|c|c|}
\hline Parameter & $\begin{array}{l}\text { Primary analysis cohort } \\
(n=257)\end{array}$ & $\begin{array}{l}\text { Sensitivity analysis cohort } \\
(n=172)\end{array}$ \\
\hline Age, years & $(n=220)$ & $(n=151)$ \\
\hline Mean (SD) & $50.2(12.3)$ & $49.9(12.6)$ \\
\hline \multicolumn{3}{|l|}{ Sex, $n / N(\%)$} \\
\hline Female & $211 / 257(82.1)$ & $142 / 172(82.6)$ \\
\hline Male & $32 / 257(12.5)$ & $23 / 172(13.4)$ \\
\hline Unknown & $14 / 257(5.4)$ & $7 / 172(4.1)$ \\
\hline \multirow[t]{2}{*}{ Years since migraine diagnosis, mean (SD) } & $(n=246)$ & $(n=168)$ \\
\hline & $5.0(5.2)$ & $5.0(5.6)$ \\
\hline \multirow[t]{2}{*}{ Years since initiation of onabotulinumtoxinA, mean (SD) } & $(n=218)$ & $(n=151)$ \\
\hline & $3.8(3.1)$ & $3.7(3.1)$ \\
\hline \multicolumn{3}{|l|}{ Index CGRP mAb treatment, $n / N(\%)$} \\
\hline Erenumab $^{\mathrm{b}}$ & $200 / 257(77.8)$ & $134 / 172(77.9)$ \\
\hline $70 \mathrm{mg}$ & $136 / 257(52.9)$ & $92 / 172(53.5)$ \\
\hline $140 \mathrm{mg}$ & $62 / 257(24.1)$ & $41 / 172(23.8)$ \\
\hline Galcanezumab $240 \mathrm{mg}^{\mathrm{c}}$ & $42 / 257(16.3)$ & $29 / 172(16.9)$ \\
\hline Fremanezumab & $15 / 257(5.8)$ & $9 / 172(5.2)$ \\
\hline $225 \mathrm{mg}$ & $8 / 257(3.1)$ & $4 / 172(2.3)$ \\
\hline $675 \mathrm{mg}$ & $7 / 257(2.7)$ & $5 / 172(2.9)$ \\
\hline
\end{tabular}

AHS American Headache Society, CGRP calcitonin gene-related peptide, HIT-6 6-item Headache Impact Test, $m A b$ monoclonal antibody, $M H D$ monthly headache day; MIDAS Migraine Disability Assessment

a The sensitivity analysis cohort was aligned to the AHS position statement's recommendation for when to initiate preventive treatment with a CGRP $\mathrm{mAb}$ [31] and included only patients with at least four MHDs at baseline and at least moderate headache-related disability (MIDAS score $>11$ or HIT-6 score $>50$ )

b Erenumab dose was not reported in two patients in the primary analysis cohort and one patient in the sensitivity analysis cohort

${ }^{c}$ Galcanezumab is administered as a $240-\mathrm{mg}$ loading dose, followed by monthly doses of $120 \mathrm{mg}$

between treatment visits ranged from 88 to 90 days, indicating that most patients attended appointments at 3-month intervals. Across visits, $96.7-99.6 \%$ of patients received onabotulinumtoxinA during the visit, and the median dose was $165 \mathrm{U}$ at baseline and at all visits during combination treatment (range across all visits: $115-200 \mathrm{U})$. Changes in the CGRP $\mathrm{mAb}$ brand were reported in $3.3-4.2 \%$ of patients, and changes in dose were reported in $7.8-18.1 \%$ of patients across visits. Treatment characteristics were similar in the AHS CGRP mAb cohort (data not shown).

\section{Safety and Tolerability}

In the primary analysis cohort, $95.3 \%(245 / 257)$ patients had at least one follow-up visit. Sixtytwo $(25.3 \%)$ of these 245 patients discontinued onabotulinumtoxinA and/or CGRP mAb at any time post-baseline (Table 3). Patients discontinued CGRP mAb $(23.3 \% \quad[57 / 245])$ 
Table 2 Headache characteristics and disability at baseline

\begin{tabular}{|c|c|c|c|c|}
\hline \multirow[t]{2}{*}{ Characteristic } & \multicolumn{2}{|c|}{ Primary analysis cohort $(n=257)$} & \multicolumn{2}{|c|}{$\begin{array}{l}\text { Sensitivity analysis cohort } \\
(n=172)\end{array}$} \\
\hline & $\begin{array}{l}\text { Before } \\
\text { onabotulinumtoxinA }\end{array}$ & $\begin{array}{l}\text { Baseline } \\
\text { (before } \\
\text { mAb) }\end{array}$ & $\begin{array}{l}\text { Before } \\
\text { onabotulinumtoxinA }\end{array}$ & $\begin{array}{c}\text { Baseline } \\
\text { (before } \\
\text { mAb) }\end{array}$ \\
\hline Headache frequency, days/month & $(n=241)$ & $(n=246)$ & $(n=163)$ & $(n=172)$ \\
\hline Mean $(S D)^{b}$ & $21(8)$ & $12(8)$ & $21(8)$ & $13(8)$ \\
\hline $\begin{array}{l}\text { Change from pre-onabotulinumtoxinA, } \\
\text { mean }(95 \% \mathrm{CI})\end{array}$ & & $\begin{array}{l}-9(-11 \\
-8)\end{array}$ & & $\begin{array}{l}-8(-10 \\
-6)\end{array}$ \\
\hline \multicolumn{5}{|l|}{ Category, $n / N(\%)$} \\
\hline$\leq 5$ & $6 / 257(2.3)$ & $\begin{array}{r}59 / 257 \\
(23.0)\end{array}$ & $3 / 172(1.7)$ & $\begin{array}{r}25 / 172 \\
(14.5)\end{array}$ \\
\hline $6-10$ & $12 / 257(4.7)$ & $\begin{array}{r}84 / 257 \\
(32.7)\end{array}$ & $9 / 172(5.2)$ & $\begin{array}{r}65 / 172 \\
(37.8)\end{array}$ \\
\hline $11-14$ & $12 / 257(4.7)$ & $\begin{array}{r}24 / 257 \\
(9.3)\end{array}$ & $11 / 172(6.4)$ & $\begin{array}{r}20 / 172 \\
(11.6)\end{array}$ \\
\hline $15-19$ & $67 / 257(26.1)$ & $\begin{array}{r}29 / 257 \\
(11.3)\end{array}$ & $44 / 172(25.6)$ & $\begin{array}{r}22 / 172 \\
(12.8)\end{array}$ \\
\hline$\geq 20$ & $138 / 257(53.7)$ & $\begin{array}{r}50 / 257 \\
(19.5)\end{array}$ & $96 / 172(55.8)$ & $\begin{array}{r}40 / 172 \\
(23.3)\end{array}$ \\
\hline Unknown & $22 / 257(8.6)$ & $\begin{array}{r}11 / 257 \\
(4.3)\end{array}$ & $9 / 172(5.2)$ & 0 \\
\hline Headache intensity $^{c}$ & NA & $(n=232)$ & NA & $(n=165)$ \\
\hline Mean (SD) & & $6.5(3.6)$ & & $6.7(4.1)$ \\
\hline MIDAS score & & $(n=215)$ & & $(n=171)$ \\
\hline Mean (SD) & NA & $43.7(43.4)$ & NA & $52.0(44.7)$ \\
\hline Moderate to very severe disability, $n / N(\%)$ & NA & $\begin{array}{r}179 / 215 \\
(83.3)\end{array}$ & NA & $\begin{array}{r}160 / 171 \\
(93.6)\end{array}$ \\
\hline HIT-6 score & & $(n=138)$ & & $(n=116)$ \\
\hline Mean (SD) & NA & $60.8(7.2)$ & NA & $61.5(6.3)$ \\
\hline Substantial to severe impact, $n / N(\%)$ & NA & $\begin{array}{r}112 / 138 \\
(81.2)\end{array}$ & NA & $\begin{array}{r}98 / 116 \\
(84.5)\end{array}$ \\
\hline PHQ-9 score & & $(n=69)$ & & $(n=56)$ \\
\hline
\end{tabular}


Table 2 continued

\begin{tabular}{|c|c|c|c|c|}
\hline \multirow[t]{2}{*}{ Characteristic } & \multicolumn{2}{|c|}{ Primary analysis cohort $(n=257)$} & \multicolumn{2}{|c|}{ Sensitivity analysis cohort ${ }^{a}(n=172)$} \\
\hline & $\begin{array}{l}\text { Before } \\
\text { onabotulinumtoxinA }\end{array}$ & $\begin{array}{c}\text { Baseline } \\
\text { (before } \\
\text { mAb) }\end{array}$ & $\begin{array}{l}\text { Before } \\
\text { onabotulinumtoxinA }\end{array}$ & $\begin{array}{c}\text { Baseline } \\
\text { (before } \\
\text { mAb) }\end{array}$ \\
\hline Mean (SD) & NA & $5.7(5.0)$ & NA & $6.8(4.8)$ \\
\hline $\begin{array}{l}\text { Moderate to severe depression, } \\
n / N(\%)\end{array}$ & NA & $\begin{array}{l}14 / 69 \\
(20.3)\end{array}$ & NA & $\begin{array}{l}14 / 56 \\
(25.0)\end{array}$ \\
\hline
\end{tabular}

AHS American Headache Society, CGRP calcitonin gene-related peptide, HIT-6 6-item Headache Impact Test, $M H D$ monthly headache day, MIDAS Migraine Disability Assessment, PHQ-9 9-item Patient Health Questionnaire, NA not available, $m A b$ monoclonal antibody

a The sensitivity analysis cohort was aligned to the AHS position statement's recommendation for when to initiate preventive treatment with a CGRP $\mathrm{mAb}$ [31] and included only patients with at least four MHDs at baseline and at least moderate headache-related disability (MIDAS score $>11$ or HIT-6 score $>50$ )

b Standardized to 30-day denominator

${ }^{c}$ Headache intensity rated on a $0-10$ scale

approximately sevenfold more often than onabotulinumtoxinA (3.3\% [8/245]). The most common reasons for CGRP mAb discontinuation were lack of insurance reimbursement (24/ 57 [42.1\%]) and lack of effect (12/57 [21.1\%]). The most common reason for discontinuing onabotulinumtoxinA was lack of reimbursement (2/8 [25.0\%]). AEs were reported by $27.8 \%$ $(68 / 245)$ of patients across all visits (Table 3$)$. The most common AE was constipation (8.6\% [21/245]), occurring most frequently in patients receiving erenumab $(18 / 21)$.

\section{Changes in Headache Frequency and Intensity (Primary Analysis Cohort)}

Compared with onabotulinumtoxinA treatment alone (baseline), adding CGRP mAb provided statistically significant and clinically meaningful reductions in mean MHDs at all visits (95\% CIs did not include 0; Fig. 3a). In the primary analysis cohort, mean decreases in MHDs compared with baseline ranged from 3.5 to 4.0 MHDs across visits occurring approximately 6,9 , and 12 months after initiation of combination therapy (Fig. 3a). Approximately one-third (31.5-36.7\%) of patients had a $\geq 50 \%$ reduction in MHDs after approximately 6 to 12 months (Fig. 3b). Data for all timepoints evaluated are shown in Fig. 3a, b. Mean headache intensity scores significantly decreased from baseline at all visits, with mean score reductions ranging from 0.4 to 0.9 points (Supplemental Figure S1).

\section{Changes in Migraine-Related Disability (Primary Analysis Cohort)}

Mean MIDAS scores significantly decreased from baseline by 6.1 to 11.1 points during approximately 6 to 12 months of combination treatment (Fig. 4a). Mean decreases in MIDAS score exceeded 5 points at all visits, indicating that the magnitude of the mean reductions was clinically meaningful at all timepoints [34]. Magnitudes of reductions were similar, but the mean reductions from baseline were not statistically significant at later visits, likely due to the reduced sample size. After approximately 6 to 12 months of combination treatment, $43.7-45.1 \%$ of patients had $\mathrm{a} \geq 5$-point reduction from baseline (Fig. 4b), and 27.1-29.6\% had $\mathrm{a} \geq 30 \%$ reduction in MIDAS score (Fig. $4 \mathrm{c}$ ). 
Table 3 Discontinuations and adverse events in the primary analysis cohort

\begin{tabular}{|c|c|c|c|c|c|}
\hline & $\begin{array}{l}\text { Visit } 1 \\
\sim 3 \text { months } \\
(n=232)\end{array}$ & $\begin{array}{l}\text { Visit } 2 \\
\sim 6 \text { months } \\
\left(n=197^{\mathrm{a}}\right)\end{array}$ & $\begin{array}{l}\text { Visit } 3 \\
\sim 9 \text { months } \\
\left(n=153^{\mathrm{a}}\right)\end{array}$ & $\begin{array}{l}\text { Visit } 4 \\
\sim 12 \text { months } \\
\left(n=118^{\mathrm{a}}\right)\end{array}$ & $\begin{array}{l}\text { Total: } \\
\text { patients with } \geq 1 \text { follow-up } \\
\text { visit } \\
(N=245)\end{array}$ \\
\hline \multicolumn{6}{|l|}{ Discontinuations, $n / N(\%)$} \\
\hline Any ${ }^{b}$ & $17 / 232(7.3)$ & $16 / 197(8.1)$ & $\begin{array}{r}16 / 153 \\
(10.5)\end{array}$ & $13 / 118(11.0)$ & $62 / 245(25.3)$ \\
\hline OnabotulinumtoxinA & $2 / 232(0.9)$ & $4 / 197(2.0)$ & $2 / 153(1.3)$ & 0 & $8 / 245(3.3)$ \\
\hline Lack of reimbursement & $1 / 2(50.0)$ & $1 / 4(25.0)$ & 0 & 0 & $2 / 8(25.0)$ \\
\hline Lack of effect & 0 & 0 & $1 / 2(50.0)$ & 0 & $1 / 8(12.5)$ \\
\hline Safety/tolerability & $1 / 2(50.0)$ & 0 & 0 & 0 & $1 / 8(12.5)$ \\
\hline Other/unknown & 0 & $3 / 4(75.0)$ & $1 / 2(50.0)$ & 0 & $4 / 8(50.0)$ \\
\hline $\begin{array}{l}\text { CGRP monoclonal } \\
\text { antibody }\end{array}$ & $17 / 232(7.3)$ & $12 / 197(6.1)$ & $15 / 153(9.8)$ & $13 / 118(11.0)$ & $57 / 245(23.3)$ \\
\hline Lack of reimbursement & $8 / 17(47.1)$ & $2 / 12(16.7)$ & $10 / 15(66.7)$ & $5 / 13(35.7)$ & $24 / 57(42.1)$ \\
\hline Lack of effect & $1 / 17(5.9)$ & $6 / 12(50.0)$ & $4 / 15(26.7)$ & $1 / 13(7.1)$ & $12 / 57(21.1)$ \\
\hline Safety/tolerability & $4 / 17(23.5)$ & $2 / 12(16.7)$ & $1 / 15(6.7)$ & $2 / 13(14.3)$ & $9 / 57(15.8)$ \\
\hline Other/unknown & $4 / 17(23.5)$ & $2 / 12(16.7)$ & 0 & $6 / 13(42.9)$ & $12 / 57(21.1)$ \\
\hline \multicolumn{6}{|l|}{$A E s, n(\%)$} \\
\hline Any AE & $35(15.1)$ & $28(14.2)$ & $22(14.4)$ & $13(11.0)$ & $68(27.8)$ \\
\hline \multicolumn{6}{|l|}{ AEs in $>2$ patients ${ }^{b}$} \\
\hline Constipation & $11(4.7)$ & $6(3.0)$ & $8(5.2)$ & $1(0.8)$ & $21(8.6)$ \\
\hline Nausea & $4(1.7)$ & $2(1.0)$ & $1(0.7)$ & $2(1.7)$ & $8(3.3)$ \\
\hline Musculoskeletal pain & $2(0.9)$ & $3(1.5)$ & $2(1.3)$ & $2(1.7)$ & $6(2.4)$ \\
\hline Neck pain & $2(0.9)$ & $1(0.5)$ & $1(0.7)$ & $3(2.5)$ & $6(2.4)$ \\
\hline Headache worsening & $2(0.9)$ & $2(1.0)$ & $1(0.7)$ & 0 & $5(2.0)$ \\
\hline Migraine worsening & $3(1.3)$ & $1(0.5)$ & 0 & 0 & $4(1.6)$ \\
\hline Erythema & 0 & 0 & $2(1.3)$ & $2(1.7)$ & $3(1.2)$ \\
\hline Injection site pain & $2(0.9)$ & $1(0.5)$ & 0 & 0 & $3(1.2)$ \\
\hline Neuropathy & 0 & $3(1.5)$ & 0 & 0 & $3(1.2)$ \\
\hline
\end{tabular}

Values are number of patients (\%)

$A E$ adverse event, CGRP calcitonin gene-related peptide

a Events reported at visits 2, 3, and 4 are not cumulative

b Discontinuation of onabotulinumtoxinA and/or CGRP antibody treatment 


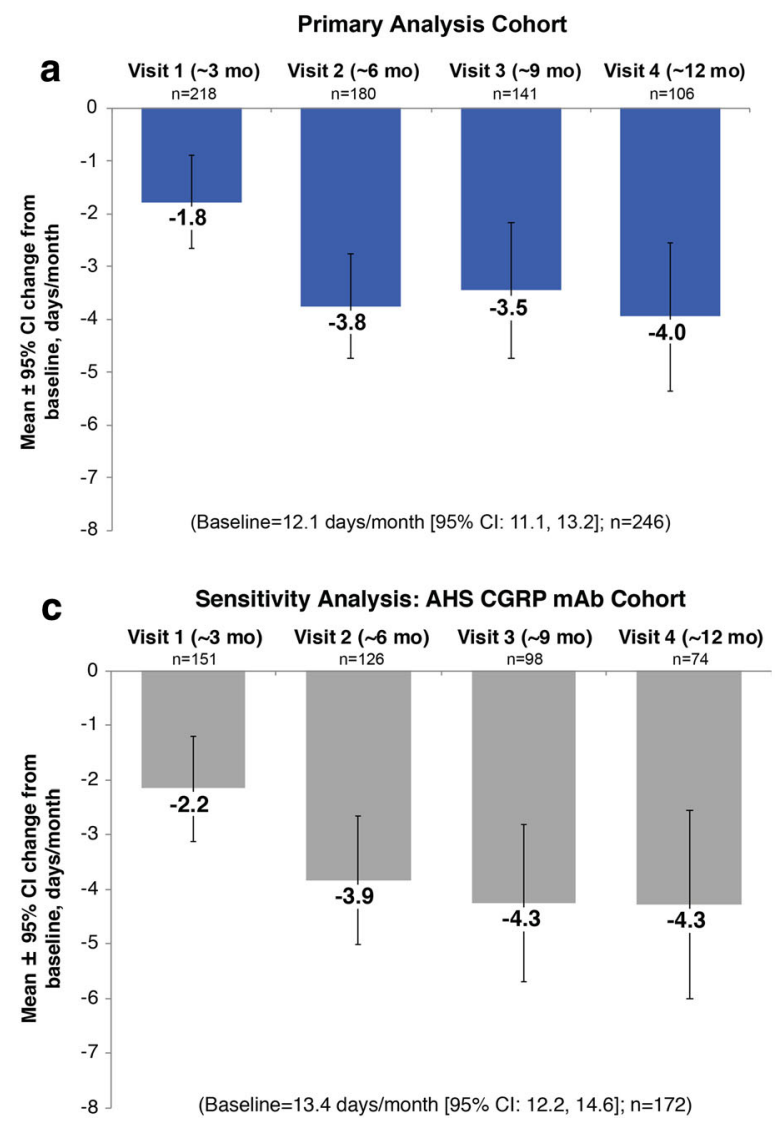

Fig. 3 Change in monthly headache frequency during combination therapy with onabotulinumtoxin $A$ and a CGRP monoclonal antibody. a Mean change from baseline and $\mathbf{b}$ percentage of patients with $\mathbf{a} \geq 50 \%$ reduction from baseline in monthly headache frequency in the primary analysis cohort. $\mathbf{c}$ Mean change from baseline

Data for all timepoints evaluated are shown in Fig. 4a-c.

\section{Outcomes in the AHS CGRP mAb Cohort (Sensitivity Analysis)}

Magnitudes of improvement in headache characteristics and migraine-related disability were similar but slightly greater in the AHS CGRP $\mathrm{mAb}$ cohort (patients with higher MHDs and at least moderate disability at baseline). In these patients, mean headache frequency of 13.4 MHDs at baseline decreased by 3.9 to 4.3 MHDs after approximately 6 to 12 months of combination therapy (Fig. 3c). Approximately one-
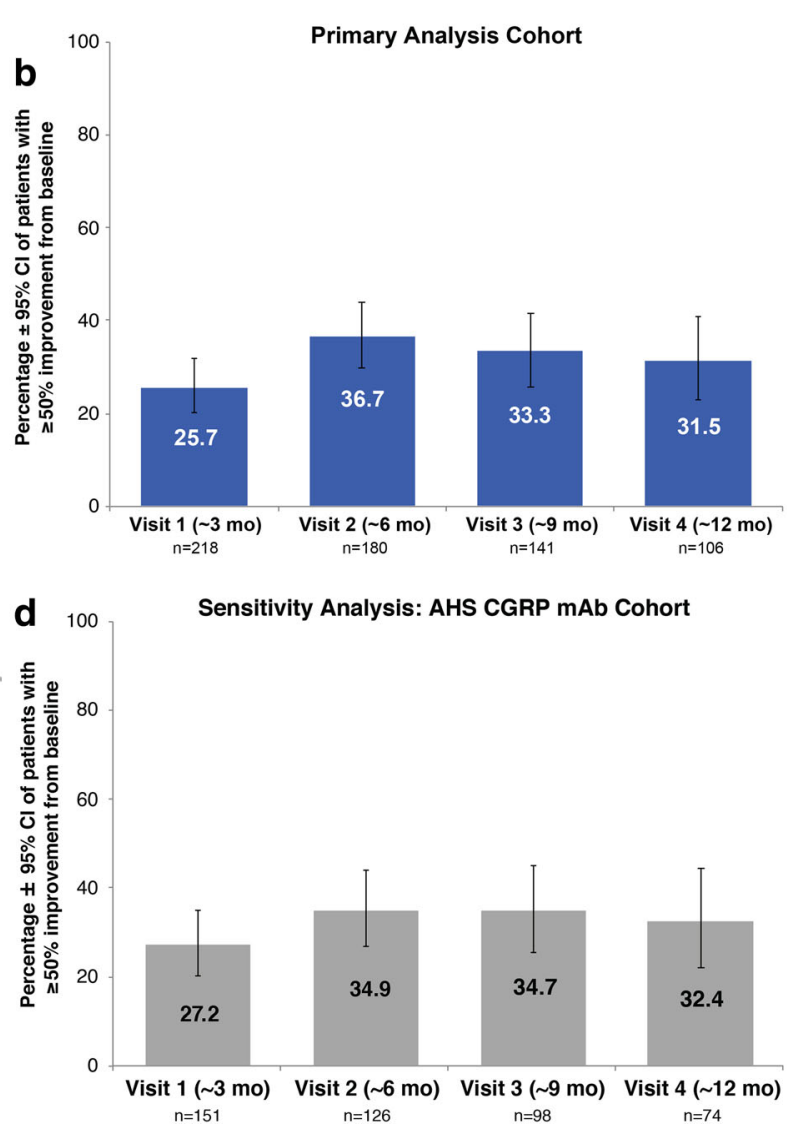

and $\mathbf{d}$ percentage of patients with a $\geq 50 \%$ reduction from baseline in monthly headache frequency in the AHS CGRP mAb cohort. AHS American Headache Society, CGRP calcitonin gene-related peptide, $C I$ confidence interval, $m A b$ monoclonal antibody

third (32.4-34.9\%) of patients had a $\geq 50 \%$ reduction in MHDs approximately 6 to 12 months after initiating CGRP mAb add-on therapy (Fig. 3d). Mean headache intensity scores significantly decreased from baseline at approximately 3, 6, and 12 months after add-on therapy, with mean score reductions ranging from 0.4 to 1.1 points (Supplemental Figure S1).

Mean MIDAS scores decreased by 8.4 to 13.7 points (Fig. 4d), 58.3-62.1\% of patients had a $\geq 5$-point reduction in MIDAS score (Fig. 4e), and $36.4-39.3 \%$ had $\mathrm{a} \geq 30 \%$ reduction in MIDAS score (Fig. 4f) after approximately 6 to 12 months of combination therapy. Patterns of discontinuations and AEs in the AHS cohort 

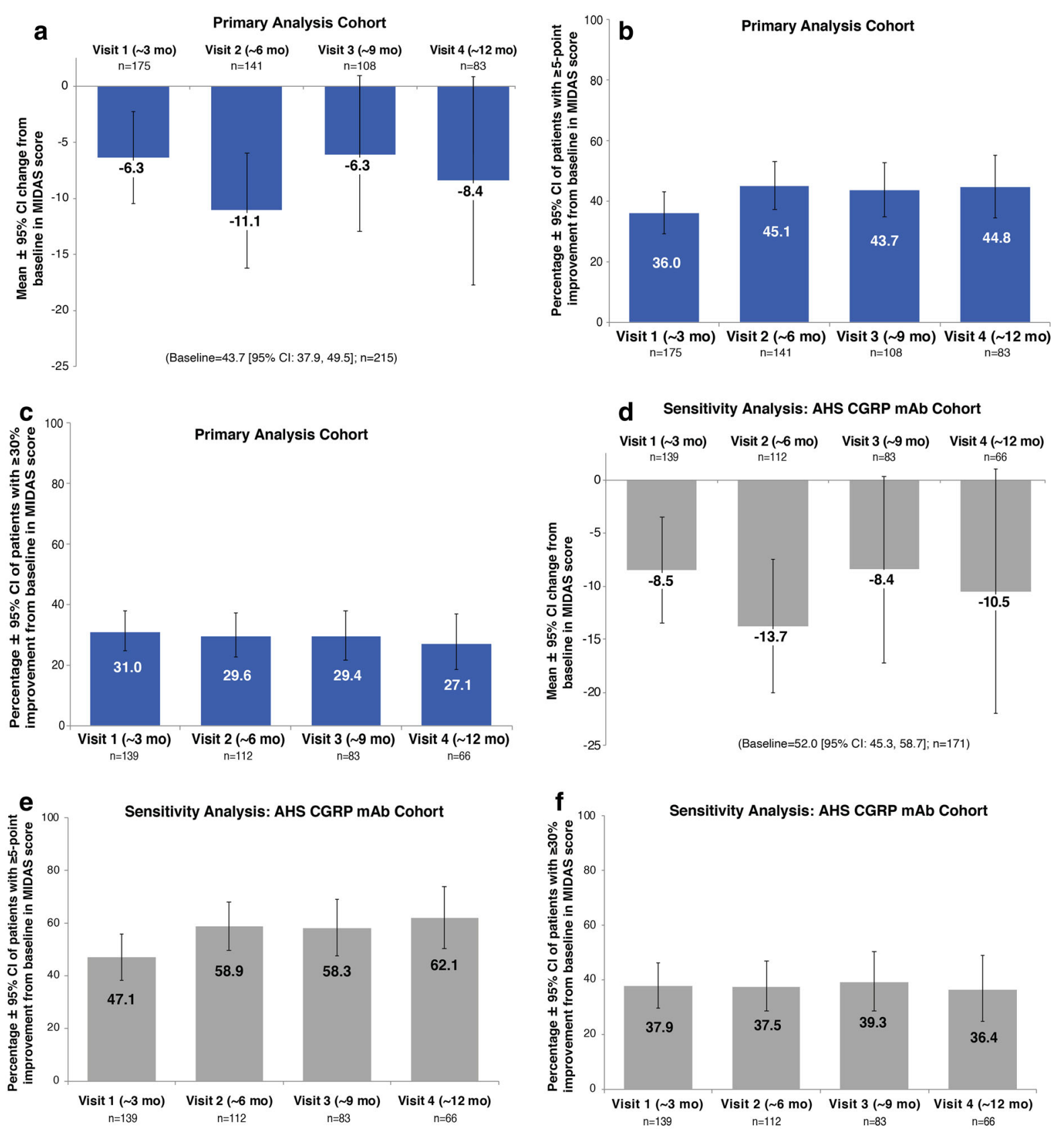

Fig. 4 Change in migraine-associated disability during combination therapy with onabotulinumtoxin $A$ and a CGRP monoclonal antibody. a Mean change from baseline in MIDAS score, $\mathbf{b}$ percentage of patients with $\mathrm{a} \geq 5$-point reduction in MIDAS score, and $\mathbf{c}$ percentage of patients with $\mathrm{a} \geq 30 \%$ improvement from baseline in MIDAS score in the primary analysis cohort. d Mean

change from baseline in MIDAS score, e percentage of patients with $a \geq 5$-point reduction in MIDAS score, and f percentage of patients with $a \geq 30 \%$ improvement from baseline in MIDAS score in the AHS CGRP mAb cohort. AHS American Headache Society, CGRP calcitonin gene-related peptide, $C I$ confidence interval, $m A b$ monoclonal antibody, $M I D A S$ migraine disability assessment 
were similar to those observed in the primary analysis cohort.

\section{DISCUSSION}

Both onabotulinumtoxinA [7-11] and CGRP mAbs [35-39] have separately established safety and efficacy for the prevention of migraine attacks in those with CM. However, combination use of onabotulinumtoxinA and CGRP mAbs may be indicated in patients who continue to experience significant migraine burden despite receiving preventive and acute treatments or who are refractory to multiple oral preventive therapies. $\mathrm{CM}$ is polygenic and involves multiple neuroanatomic pathways, multiple vasoactive neuropeptides, and many different receptors [40]. A monotherapy approach is unlikely to achieve optimal management in some patients. The ultimate aspirational goal of migraine treatment is freedom from the impact of migraine, or migraine freedom, a concept that can vary greatly among individuals, with some people considering themselves migraine-free if they have an occasional migraine attack, as long as it does not impact their daily activities, whereas others may expect complete freedom from any headache and associated symptoms. These goals, despite being aspirational, are important considerations for clinicians treating patients with $\mathrm{CM}$ and should guide treatment decisions. Therefore, a layered approach that considers the patient's treatment goals and targets different pathways involved in migraine pathophysiology may be beneficial [20]. Many clinicians add CGRP mAbs to onabotulinumtoxinA treatment in $\mathrm{CM}$ patients in their practices [25, 41]. However, data on the safety and efficacy of combination use are limited. Preclinical and clinical data suggest that combination treatment could be additive or synergistic, as CGRP mAbs and onabotulinumtoxinA have distinct mechanisms of action in the prevention of migraine [12-15, 21, 22, 28].

In this retrospective, real-world study of 257 patients receiving onabotulinumtoxinA combined with either erenumab, galcanezumab, or fremanezumab, treatment was generally well tolerated and no new safety signals were identified. Patients discontinued CGRP mAb more frequently than onabotulinumtoxinA (23.3 vs. $3.3 \%)$. However, all patients received at least two onabotulinumtoxinA treatments before initiating combination treatment, possibly contributing to the lower discontinuation rate for onabotulinumtoxinA, as patients not tolerating were likely precluded from study participation. Safety concerns were not the primary driver of CGRP mAb discontinuation, as most patients discontinued because of a lack of insurance reimbursement (42\%) or a lack of effect $(21 \%)$.

The safety profile during combination treatment was similar to that observed in prior analyses of each treatment alone. The only $\mathrm{AE}$ reported by $\geq 5 \%$ of patients was constipation, which is reported with erenumab [17], the CGRP mAb prescribed to $77 \%$ of patients in this study. In randomized phase 3 clinical trials, the only AEs reported in $\geq 2 \%$ of participants treated with erenumab $70 \mathrm{mg}$ and at an incidence $\geq 2 \%$ greater than placebo were injection-site reactions (6\%) [17]. The incidence of constipation was higher (34\%) in a real-world observational study of $158 \mathrm{CM}$ patients treated with erenumab with or without other preventives or onabotulinumtoxinA [24]. Gastrointestinal and other AEs are less common with galcanezumab [38, 39] and fremanezumab $[42,43]$, occurring at rates similar to those observed with placebo in clinical trials. The most common AEs reported with onabotulinumtoxinA in placebo-controlled $\mathrm{CM}$ trials were neck pain $(9 \%)$ and headache $(5 \%)[7,8]$.

Patients included in this study had clinically meaningful responses to onabotulinumtoxinA prior to adding the CGRP $m A b$, experiencing an average decrease of 9 MHDs during onabotulinumtoxinA treatment alone. This level of benefit is consistent with results of the phase 3 PREEMPT studies, where average decreases of 7.8 to 9.0 MHDs were observed after 6 months of onabotulinumtoxinA treatment [7, 8, 44]. However, due to the nature and severity of CM, the patients in our study continued to experience an average of 12 MHDs when CGRP mAb was initiated. Combination treatment with the two therapies was associated with additional 
reductions in headache frequency, headache intensity, and migraine-related disability compared with onabotulinumtoxinA without the additive treatment. Monthly headache frequency significantly decreased from baseline at all visits, with mean reductions of approximately 3 to 4 days per month after approximately 6 to 12 months of combination treatment, and clinically meaningful reductions in migraine-related disability $(\geq 5$-point decrease in mean MIDAS score) were also observed at all visits. There are some important caveats to these findings: we could not determine when patients administered their first CGRP mAb injection, as compliance following prescribing was not assessed. Additionally, many patients did not have visits after approximately 6 months. Therefore, assessments at each visit may not accurately reflect $3,6,9$, or 12 months of combination use.

Patients in this study initiated CGRP mAbs during the first year following US FDA approval, a time period when free product was widely available. Thus, some patients may not reflect the population most likely to be prescribed this type of preventive combination treatment in the future. Therefore, we conducted a sensitivity analysis to evaluate outcomes in a population more reflective of current real-world prescribing practices and consistent with the AHS position statement [31]. The sensitivity analysis showed that despite having higher headache frequency and related disability at baseline, these patients had greater reductions in headache frequency and disability during combination treatment than did the primary analysis cohort (Figs. 3, 4).

Baseline headache burden at initiation of combination therapy in this study was consistent with that observed in CGRP mAb studies of patients who failed two or more prior preventive treatments [45-47]. In these studies, the average decrease in migraine days after 3 months of treatment was 1.8 days with monthly erenumab $70 \mathrm{mg}$ [48], 1.8 to 3.5 days with monthly erenumab $140 \mathrm{mg}[46,48]$, 4.1 days with monthly fremanezumab $225 \mathrm{mg}$ [45], and 4.1 days with monthly galcanezumab $120 \mathrm{mg}$ (with 240-mg loading dose) [47]. Three retrospective single-site studies reported mean reductions in MHDs of 4.3 to 8.1 days/month after CGRP $\mathrm{mAb}$ initiation in patients already receiving onabotulinumtoxinA [23, 25, 28]. Another real-world observational study of 158 patients with $\mathrm{CM}$ who failed at least three prior preventive drugs reported that $65 \%$ of patients treated with erenumab added to onabotulinumtoxinA had reductions in MHDs after four antibody injections, versus $26 \%$ of those treated with erenumab alone and $15 \%$ of those who received erenumab added to an oral preventive [24].

This real-world chart review has several limitations, including the fact that many patients had missing data and the reasons for adding a CGRP mAb treatment were not uniform across patients. As stated above, data represent the first year of launch of CGRP mAbs. Therefore, the distribution of different antibody brands depended on the time of product release and the way in which they were used. Because erenumab was the first FDA-approved treatment, most patients (53\%) received the $70 \mathrm{mg}$ dose. As practice evolved, more patients received the 140-mg dose potentially due to improving efficacy; the depth of response may have been better if more patients had received this dose. Insufficient numbers of patients were treated with galcanezumab and fremanezumab to allow for comparisons between different CGRP mAb groups. This is an important area for future research. Compliance with CGRP mAb injections is unknown, prohibiting confirmation of the timing of the combination treatment initiation. OnabotulinumtoxinA treatment is not always administered per label, and some variation in the timing of injections was likely. Therefore, consistency of treatment with both onabotulinumtoxinA and CGRP mAbs may have varied between patients. Lastly, the study outcome measures were not consistently reported, and reporting methods may have varied.

\section{CONCLUSIONS}

These real-world data demonstrated that combination use of onabotulinumtoxinA and a CGRP mAb was generally well tolerated, with no new safety signals identified, and associated 
with clinically meaningful improvements in headache frequency and migraine-related disability compared with onabotulinumtoxinA without CGRP mAb therapy. Additional realworld and controlled trials should be considered to further assess safety and quantify the additive or synergistic benefit of this multimodal treatment paradigm for people with CM.

\section{ACKNOWLEDGMENTS}

The authors thank and acknowledge their committed patients as well as the staff who did all of the data extraction from the patient charts.

Funding. Sponsorship for this study and Rapid Service Fee were funded by AbbVie, Irvine, CA.

Medical Writing and Editorial Assistance. Medical writing and editorial support were provided by Lela Creutz, PhD and Sean Sheffler-Collins, PhD of Peloton Advantage, LLC, an OPEN Health company, Parsippany, NJ, and was funded by AbbVie.

Authorship. All named authors meet the International Committee of Medical Journal Editors (ICMJE) criteria for authorship for this article, take responsibility for the integrity of the work as a whole, and have given their approval for this version to be published.

Authors' Contributions. AMB, AMA, and LY conceptualized and designed the study, and contributed to analysis and interpretation of the data and the review of the manuscript, revising it critically for important intellectual content. AMB, BMF, JDS, AI, and GS contributed to acquisition, analysis, and interpretation of the data, and contributed to review of the manuscript, revising it critically for important intellectual content. All authors approved the final version of the manuscript as submitted and agree to be accountable for all aspects of the work in ensuring that questions related to the accuracy or integrity of any part of the work are appropriately investigated and resolved.

Prior Presentation. This manuscript is based on work that has been previously presented at the Migraine Trust Virtual Symposium, October 3-9, 2020.

Disclosures. Andrew M. Blumenfeld, MD has served on advisory boards for AbbVie, Amgen, Alder, Teva, Supernus, Promius, Eaglet, and Lilly; and has received funding for speaking from AbbVie, Amgen, Pernix, Supernus, Depomed, Avanir, Promius, Teva, and Eli Lilly and Company. Benjamin M. Frishberg, MD has received compensation for speaking from Teva, Lilly, Biohaven, Amgen, Novartis, and AbbVie; and has served on advisory boards for Lundbeck, Upsher-Smith, and Theranica. Jack D. Schim, MD has served on advisory boards for Aeon, Allergan, Amgen, Biohaven, electroCore, Impel, Lilly, Lundbeck, Novartis, Promius, Revance, Teva, and Upsher-Smith; and has received compensation for speaking from Allergan, Amgen, Biohaven, electroCore, Lilly, Lundbeck, Novartis, Promius, Teva, and UpsherSmith. Ashley Iannone, MPH declares that there is no conflict of interest. Gary Schneider, MSPH, ScD declares that there is no conflict of interest. Larisa Yedigarova, MD, PhD and Aubrey Manack Adams, PhD are employees of AbbVie and may hold AbbVie stock.

Compliance with Ethics Guidelines. The study was conducted in accordance with International Council for Harmonisation guidelines and local legal requirements, and complied with the ethical principles of the World Medical Assembly. The New England Independent Review Board approved the study protocol and case report form (CRF) before study initiation and determined that the study had minimal risk and met requirements for a consent waiver.

Data Availability. The datasets generated and/or analyzed during the current study are available from the corresponding author on reasonable request. 
Open Access. This article is licensed under a Creative Commons Attribution-NonCommercial 4.0 International License, which permits any non-commercial use, sharing, adaptation, distribution and reproduction in any medium or format, as long as you give appropriate credit to the original author(s) and the source, provide a link to the Creative Commons licence, and indicate if changes were made. The images or other third party material in this article are included in the article's Creative Commons licence, unless indicated otherwise in a credit line to the material. If material is not included in the article's Creative Commons licence and your intended use is not permitted by statutory regulation or exceeds the permitted use, you will need to obtain permission directly from the copyright holder. To view a copy of this licence, visit http://creativecommons.org/licenses/by$\mathrm{nc} / 4.0 /$.

\section{REFERENCES}

1. IHS classification ICHD-3: migraine. 2019. https:// ichd-3.org/1-migraine/. Accessed 18 Feb 2020.

2. Lipton RB, Manack Adams A, Buse DC, Fanning KM, Reed ML. A comparison of the chronic migraine epidemiology and outcomes (CaMEO) study and American migraine prevalence and prevention (AMPP) study: demographics and headache-related disability. Headache. 2016;56:1280-9.

3. Blumenfeld AM, Varon SF, Wilcox TK, et al. Disability, HRQoL and resource use among chronic and episodic migraineurs: results from the International Burden of Migraine Study (IBMS). Cephalalgia. 2011;31:301-15.

4. Buse DC, Manack Adams A, Serrano D, Turkel C, Lipton RB. Sociodemographic and comorbidity profiles of chronic migraine and episodic migraine sufferers. J Neurol Neurosurg Psychiatry. 2010;81: 428-32.

5. Buse DC, Fanning KM, Reed ML, et al. Life with migraine: effects on relationships, career, and finances from the chronic migraine epidemiology and outcomes (CaMEO) study. Headache. 2019;59: 1286-99.

6. Lanteri-Minet M, Duru G, Mudge M, Cottrell S. Quality of life impairment, disability and economic burden associated with chronic daily headache, focusing on chronic migraine with or without medication overuse: a systematic review. Cephalalgia. 2011;31:837-50.

7. Aurora SK, Dodick DW, Turkel CC, et al. OnabotulinumtoxinA for treatment of chronic migraine: results from the double-blind, randomized, placebo-controlled phase of the PREEMPT 1 trial. Cephalalgia. 2010;30:793-803.

8. Diener HC, Dodick DW, Aurora SK, et al. OnabotulinumtoxinA for treatment of chronic migraine: results from the double-blind, randomized, placebo-controlled phase of the PREEMPT 2 trial. Cephalalgia. 2010;30:804-14.

9. Blumenfeld AM, Stark RJ, Freeman MC, Orejudos A, Manack Adams A. Long-term study of the efficacy and safety of OnabotulinumtoxinA for the prevention of chronic migraine: COMPEL study. J Headache Pain. 2018;19:13.

10. Rothrock JF, Manack Adams A, Lipton RB, et al. FORWARD study: evaluating the comparative effectiveness of onabotulinumtoxinA and topiramate for headache prevention in adults with chronic migraine. Headache. 2019;59:1700-13.

11. Ahmed F, Gaul C, Garcia-Monco JC, Sommer K, Martelletti P. An open-label prospective study of the real-life use of onabotulinumtoxinA for the treatment of chronic migraine: the REPOSE study. J Headache Pain. 2019;20:26.

12. Burstein R, Blumenfeld AM, Silberstein SD, Manack Adams A, Brin MF. Mechanism of action of onabotulinumtoxinA in chronic migraine: a narrative review. Headache. 2020;60:1259-72.

13. Durham PL. CGRP-receptor antagonists-a fresh approach to migraine therapy? N Engl J Med. 2004;350:1073-5.

14. Joussain C, Le Coz O, Pichugin A, et al. Botulinum neurotoxin light chains expressed by defective herpes simplex virus type- 1 vectors cleave SNARE proteins and inhibit CGRP release in rat sensory neurons. Toxins (Basel). 2019;11:123.

15. Cernuda-Morollón E, Ramón C, Martínez-Camblor P, Serrano-Pertierra E, Larrosa D, Pascual J. OnabotulinumtoxinA decreases interictal CGRP plasma levels in patients with chronic migraine. Pain. $2015 ; 156: 820-4$.

16. Edvinsson L, Haanes KA, Warfvinge K, Krause DN. CGRP as the target of new migraine therapiessuccessful translation from bench to clinic. Nat Rev Neurol. 2018;14:338-50. 
17. Aimovig [package insert]. Thousand Oaks, CA, and East Hanover, NJ: Amgen Inc., and Novartis Pharmaceuticals Corporation; 2020.

18. Ajovy [package insert]. North Wales, PA: Teva Pharmaceuticals USA, Inc.; 2020.

19. Emgality [package insert]. Indianapolis, IN: Eli Lilly and Company; 2019.

20. Pellesi L, Do TP, Ashina H, Ashina M, Burstein R. Dual therapy with anti-CGRP monoclonal antibodies and botulinum toxin for migraine prevention: is there a rationale? Headache. 2020;60: 1056-65.

21. Melo-Carrillo A, Strassman AM, Nir RR, et al. Fremanezumab-A humanized monoclonal anti-CGRP antibody-inhibits thinly myelinated (A $\delta)$ but not unmyelinated (C) meningeal nociceptors. J Neurosci. 2017;37:10587-96.

22. Melo-Carrillo A, Strassman AM, Schain AJ, et al. Exploring the effects of extracranial injections of botulinum toxin type $\mathrm{A}$ on prolonged intracranial meningeal nociceptors responses to cortical spreading depression in female rats. Cephalalgia. 2019;39:1358-65.

23. Armanious M, Khalil N, Lu Y, Jimenez-Sanders R. Erenumab and onabotulinumtoxinA combination therapy for the prevention of intractable chronic migraine without aura: a retrospective analysis. J Pain Palliat Care Pharmacother. 2020. https://doi. org/10.1080/15360288.2020.1829249.

24. Boudreau GP. Treatment of chronic migraine with erenumab alone or as an add on therapy: a realworld observational study. Anesth Pain Res. 2020;4: $1-4$.

25. Cohen F, Armand C, Vollbracht S. Efficacy and tolerability of CGRP monoclonal antibody medications in patients with chronic migraine undergoing treatment with onabotulinumtoxinA [poster]. Annual Meeting of the American Headache Society; 2020 June 13, 2020.

26. Gottschalk PC, Henn K, Robinson J, Schobel VR. Anti-CGRP class reduces migraine burden regardless of concomitant therapies in US clinical practice [poster]. Annual Meeting of the American Academy of Neurology; 2020.

27. Suri H, Nandyala A, Dougherty C, Ailani J. Combination of erenumab and onabotulinum toxin $\mathrm{A}$ is a safe and effective treatment for chronic migraine [poster]. Annual Meeting of the American Headache Society; 2020 June 13, 2020.

28. Singh S, Singh H, Govindarajan R. Galcanezumab in the prevention of chronic migraine in patients on botulinum toxin therapy [abstract]. Neurology. 2020;94(15 suppl):5257.

29. Robblee J, Devick KL, Mendez N, Potter J, Slonaker J, Starling AJ. Real-world patient experience with erenumab for the preventive treatment of migraine. Headache. 2020;60:2014-25.

30. Ozudogru SN, Bartell JW, Yuan H, Digre KB, Baggaley SK. The effect of adding calcitonin gene-related peptide monoclonal antibodies to onabotulinum toxin A therapy on headache burden: a retrospective observational case series. Headache. 2020;60:1442-3.

31. American Headache Society. The American Headache Society position statement on integrating new migraine treatments into clinical practice. Headache. 2019;59:1-18.

32. Kroenke K, Spitzer RL, Williams JB. The PHQ-9: validity of a brief depression severity measure. J Gen Intern Med. 2001;16:606-13.

33. Stewart WF, Lipton RB, Dowson AJ, Sawyer J. Development and testing of the Migraine Disability Assessment (MIDAS) Questionnaire to assess headache-related disability. Neurology. 2001;56:S20-8.

34. Lipton RB, Desai P, Sapra S, Buse DC, Fanning KM, Reed ML. How much change in headache-related disability is clinically meaningful? Estimating minimally important difference (MID) or change in MIDAS using data from the AMPP study [abstract PF52]. Headache. 2017;57:165-6.

35. Goadsby PJ, Reuter U, Hallstrom Y, et al. A controlled trial of erenumab for episodic migraine. N Engl J Med. 2017;377:2123-32.

36. Dodick DW, Ashina M, Brandes JL, et al. ARISE: a Phase 3 randomized trial of erenumab for episodic migraine. Cephalalgia. 2018;38:1026-37.

37. Tepper S, Ashina M, Reuter U, et al. Safety and efficacy of erenumab for preventive treatment of chronic migraine: a randomised, double-blind, placebo-controlled phase 2 trial. Lancet Neurol. 2017;16:425-34.

38. Detke HC, Goadsby PJ, Wang S, Friedman DI, Selzler KJ, Aurora SK. Galcanezumab in chronic migraine: the randomized, double-blind, placebocontrolled REGAIN study. Neurology. 2018;91: e2211-21.

39. Stauffer VL, Dodick DW, Zhang Q, Carter JN, Ailani J, Conley RR. Evaluation of galcanezumab for the prevention of episodic migraine: the EVOLVE-1 randomized clinical trial. JAMA Neurol. 2018;75: 1080-8. 
40. Pietrobon D, Moskowitz MA. Pathophysiology of migraine. Annu Rev Physiol. 2013;75:365-91.

41. Armanious M, Khalil N, Lu Y, Jimenez-Sanders R. A retrospective analysis to evaluate the response of the addition of erenumab to onabotulinumtoxinA for the prevention of intractable chronic migraine without aura [poster]. Annual Meeting of the American Headache Society; 2019 July 11-14, 2019; Philadelphia, PA.

42. Dodick DW, Silberstein SD, Bigal ME, et al. Effect of fremanezumab compared with placebo for prevention of episodic migraine: a randomized clinical trial. JAMA. 2018;319:1999-2008.

43. Silberstein SD, Dodick DW, Bigal ME, et al. Fremanezumab for the preventive treatment of chronic migraine. N Engl J Med. 2017;377:2113-22.

44. Dodick DW, Turkel CC, DeGryse RE, et al. OnabotulinumtoxinA for treatment of chronic migraine: pooled results from the double-blind, randomized, placebo-controlled phases of the PREEMPT clinical program. Headache. 2010;50:921-36.
45. Ferrari MD, Diener HC, Ning $X$, et al. Fremanezumab versus placebo for migraine prevention in patients with documented failure to up to four migraine preventive medication classes (FOCUS): a randomised, double-blind, placebo-controlled, phase 3b trial. Lancet. 2019;394:1030-40.

46. Reuter U, Goadsby PJ, Lanteri-Minet M, et al. Efficacy and tolerability of erenumab in patients with episodic migraine in whom two-to-four previous preventive treatments were unsuccessful: a randomised, double-blind, placebo-controlled, phase 3b study. Lancet. 2018;392:2280-7.

47. Mulleners W, Kim B, Lainez M, et al. A randomized, placebo-controlled study of galcanezumab in patients with treatment-resistant migraine: doubleblind results from the CONQUER study [abstract]. Neurology. 2020;94:162.

48. Goadsby PJ, Paemeleire K, Broessner G, et al. Efficacy and safety of erenumab (AMG334) in episodic migraine patients with prior preventive treatment failure: a subgroup analysis of a randomized, double-blind, placebo-controlled study. Cephalalgia. 2019;39:817-26. 